\title{
Design of Signal Timing Plan for Urban Signalized Networks including Left Turn Prohibition
}

\author{
Qinrui Tang $\mathbb{D}^{1,2}$ and Bernhard Friedrich ${ }^{2}$ \\ ${ }^{1}$ Institute of Transportation Systems, German Aerospace Center, Berlin, Germany \\ ${ }^{2}$ Institute of Transportation and Urban Engineering, Technische Universität Braunschweig, Braunschweig, Germany \\ Correspondence should be addressed to Qinrui Tang; qinruitang@gmail.com
}

Received 2 January 2018; Revised 9 April 2018; Accepted 29 April 2018; Published 27 June 2018

Academic Editor: Wai Yuen Szeto

Copyright (c) 2018 Qinrui Tang and Bernhard Friedrich. This is an open access article distributed under the Creative Commons Attribution License, which permits unrestricted use, distribution, and reproduction in any medium, provided the original work is properly cited.

\begin{abstract}
Urban road networks may benefit from left turn prohibition at signalized intersections regarding capacity, for particular traffic demand patterns. The objective of this paper is to propose a method for minimizing the total travel time by prohibiting left turns at intersections. With the flows obtained from the stochastic user equilibrium model, we were able to derive the stage generation, stage sequence, cycle length, and the green durations using a stage-based method which can handle the case that stages are sharing movements. The final output is a list of the prohibited left turns in the network and a new signal timing plan for every intersection. The optimal list of prohibited left turns was found using a genetic algorithm, and a combination of several algorithms was employed for the signal timing plan. The results show that left turn prohibition may lead to travel time reduction. Therefore, when designing a signal timing plan, left turn prohibition should be considered on a par with other left turn treatment options.
\end{abstract}

\section{Introduction}

Left turns at signalized intersections may cause efficiency problems, because they have comparatively high potential for conflicts with other movements. When permitted left turns are used, the delay of vehicles is determined both by the traffic signal and by the opposing vehicles. As a result, vehicles turning left would usually wait longer than other movements. Protected left turns can be applied at the expense of increasing the intergreen time and decreasing the effective green time, resulting in efficiency problems. Therefore, to improve efficiency, left turn prohibition (LTP) is investigated in urban networks as a remedy for urban congestion problems.

Previous left turn treatment guidelines provide the treatment of permitted/protected left turns at signalized intersections [1-6], while very few refer to left turn prohibition. Although left turns can be avoided by constructing unconventional intersections such as U-turns in the middle of roads [7-12], mid-block left turns [13, 14], continuous flow intersections, jughandle intersections [15], exit lanes for left turn [16], special width intersection [17], and tandem intersection/presignals [18-20], due to space limitation in cities and high construction expense, these unconventional intersections may not be feasible in the urban area. The impact of these unconventional intersections on networks is also not clear because the intersections/locations are usually studied in isolation. Another alternative to left turn prohibition, which does not necessitate infrastructure alteration, would be to redesign the network. By redesigning a network in this way, flow redistribution can be obtained using traffic assignment models [21-24]. However, when the turning restriction problem is formulated as a pure network design problem, signal settings are ignored. Consequently, this is making it unsuitable for the use in the urban signalized networks. In these environments, it is necessary to design signal timing plan for the LTP problem.

The methods for signal timing plan design are the stage-based method [25-29], the group-based method [3032], and the lane-based method [33-35]. The stage-based method, whose cycle is composed of stages, determines green durations of each stage by minimizing delays or other objectives. In practice, all existing methods for network signal setting design follow the stage-based method [29]. The most famous signal control method using the stage-based method 


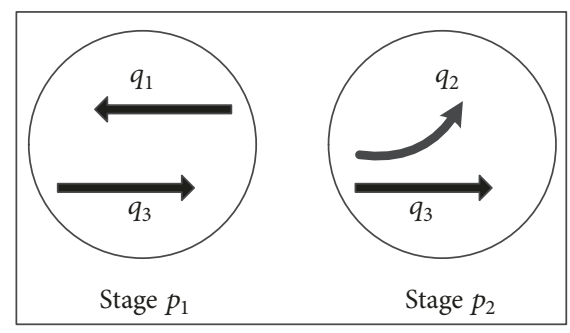

FIGURE 1: An example of two stages sharing movements [38].

is TRANSYT. However, the existing stage-based methods rarely include the stages sharing movements when generating stages and optimizing/calculating signal timing. The groupbased method and the lane-based method have a similar formulation. The difference is that the lane-based method can also assign lane markings. The lane-based method was used in turning restriction problems [36] and was integrated with traffic assignment models [37], but both methods have not been practically applied. In consideration of the practical extension, the stage-based method is used in this paper. The research gap filled for the stage-based is to explain the treatment of the case that stages are sharing movements (see Figure 1).

This paper presents a method for selecting left turns with a genetic algorithm for prohibition and designing a fixed signal timing plan including LTP for a time in a day, by minimizing total travel times. After prohibiting left turns, the proposed method adjusts signal settings using the stagebased method. Once the selected left turns are prohibited, all stages are regenerated and then the stage sequence is optimized. Besides, cycle length and green duration are calculated. The intersections in networks are coordinated by the common cycle length, which is the largest cycle length of all intersections. Stochastic user equilibrium (SUE) is integrated for forecasting redistributed traffic flows, and it is used for the evaluation of the LTP. To test the proposed method, one should set the total travel time without LTP as a base case and compare it with the total travel time with LTP.

The following abbreviations are used in this paper: LTP represents left turn prohibition; SUE means stochastic user equilibrium; OD indicates Origin-Destination; TSP stands for traveling salesman problem.

\section{Methodology}

2.1. Overall. The methodology consists of three parts: selecting the prohibited left turns, signal timing plan design including permitted/protected/prohibited left turns for each intersection, and an SUE used for a realistic assignment of the demand.

The flowchart in Figure 2 presents the approach in a chronological sequence. LTP influences the network link flows. The link flows are estimated using SUE based on a link cost function, which does not consider signal timing. Then for each left turn that is not prohibited it is decided whether it is permitted or protected. Feasible stages are generated, and then the signal sequence is determined by minimizing the

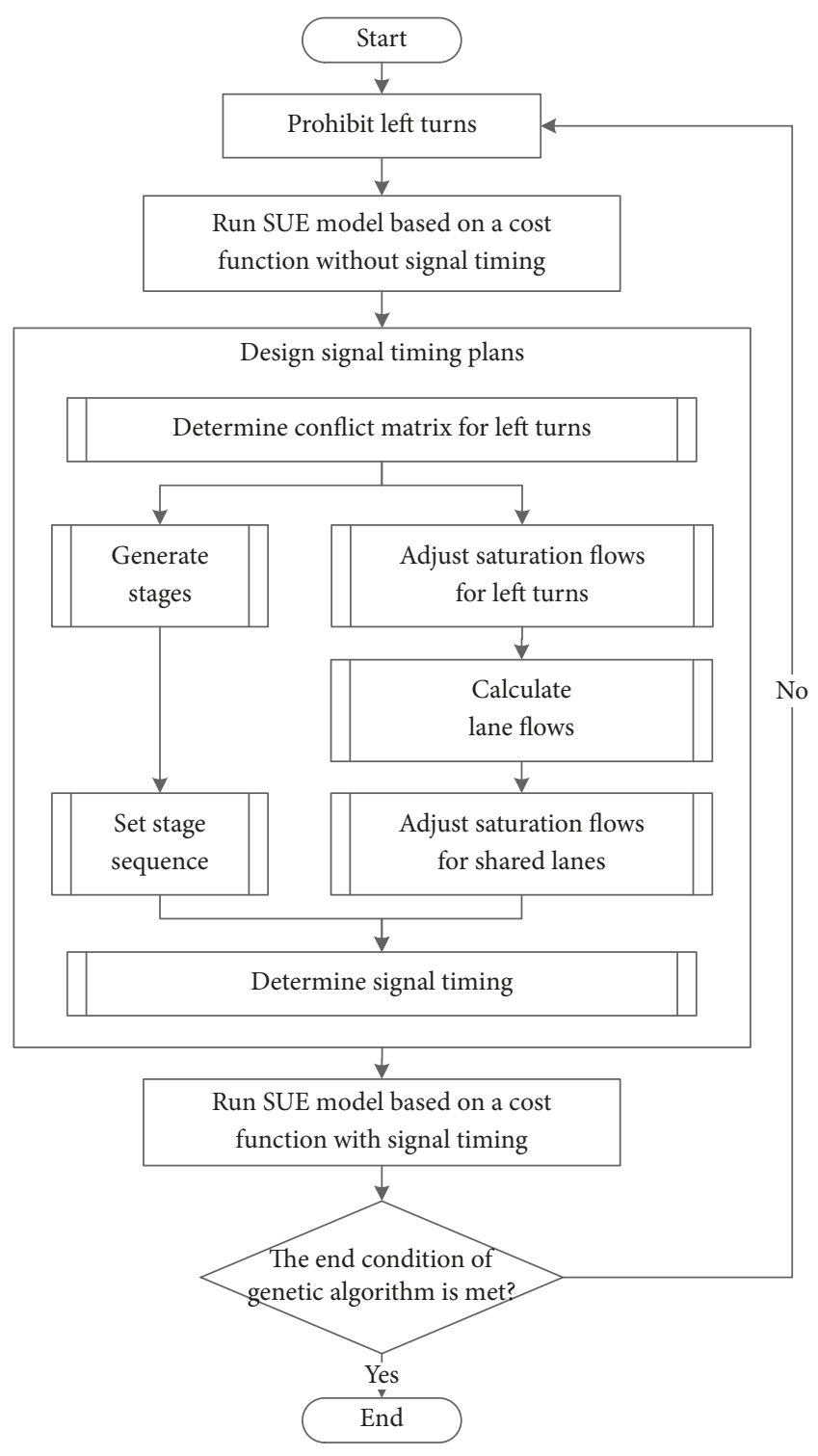

FIGURE 2: Flowchart of signal timing plan design including left turn prohibition.

total intergreen time. In parallel, lane flows are calculated, and saturation flows are adjusted to determine signal timing. The SUE model is run once again using a cost function based on signal timing. This is performed to evaluate the total travel time with the LTP and the signal timing. Left turns are selected to be prohibited with genetic algorithms. These processes will be repeated until all selected left turns are tested.

The following sections explain each processing box and subprocessing step of the flowchart in detail.

2.2. Left Turn Prohibition. Each network includes many left turns. Each left turn has two possible states: it can be either allowed or prohibited. An LTP combination is defined as the decision regarding the state of each left turn in the network. Considering the number of left turns in the network, the total number of LTP combinations can be very large. Therefore, 
using the algorithm detailed in Section 3, we reduced the number of possible LTP combinations.

The overall objective is to minimize the total travel time in the network as described in (1). This equation sums the total travel time, separating left turn links from other links. The total travel time without LTP is the reference case. Only if the total travel time with LTP is smaller than the reference total travel time can the LTP combination be applied. Eventually, the LTP combination with the minimal total travel time is selected as the solution.

For each selected prohibited left turn, we assumed that the lane would be used for through movement. We also made sure that each selected left turn can be prohibited. Specifically, a check was performed that the prohibition of this turn would still allow network connectivity. Thus each OD path set includes at least one path (see (2)). Additionally, the number of through exit lanes is greater than or equal to the number of through approaching lanes (see (3)). If both of these constraints cannot be met, the left turn is not prohibited.

$$
\begin{aligned}
\min & \text { TT } \\
= & \sum_{a \in M / L} q_{a} t_{a}\left(q_{a}, \eta\right) \\
& +\sum_{a \in L}\left(1-x_{a}\right) q_{a} t_{a}\left(q_{a}, \eta\right)
\end{aligned}
$$

subject to $K_{o d} \neq \emptyset, \quad \forall o \in O, d \in D$

$$
\begin{aligned}
N_{\alpha, z, i} \leq & N_{\epsilon, z, i}, \\
& \forall z=1, \ldots, N_{Z}, i=1, \ldots, N_{A, z},
\end{aligned}
$$

where TT is total travel time (h); $M$ is set of links; $L$ is set of left turn links; $q_{a}, \forall a \in M$, is flow of link $a$ (veh/h); $t_{a}\left(q_{a}, \eta\right), \forall a \in M$, is travel time of link $a(\mathrm{~h}) ; \eta$ is signal settings defined as stage, stage sequence, cycle length, and green durations; $x_{a}, \forall a \in L$, is indicator of prohibited left turns: $x_{a}=1$, if the left turn is prohibited and $x_{a}=0$, otherwise; $O$ is origin set; $D$ is destination set; $K_{o d}$ is path set between origin $o$ and destination $d$; $z$ is intersection index; $N_{Z}$ is number of intersections in the network; $i$ is arm index; $N_{A, z}$ is the number of arms at intersection $z ; N_{\alpha, z, i}$ is the number of approaching lanes for through movements in arm $i$ at intersection $z ; N_{\epsilon, z, i}$ is the number of exit lanes for through movements in $\operatorname{arm} i$ at intersection $z$.

2.3. Stochastic User Equilibrium Model. A traffic assignment model describes how traffic flows are distributed in the network. In SUE, one of the traffic assignment models, drivers choose routes with minimal perceived travel time. The relevant link flows change according to the result of path choice and influence the travel time. Drivers iteratively react to updated travel time until any change of routes makes the perceived travel time longer.

Link flow calculation and travel time estimation are critical steps in SUE. Link flow is calculated by the product of OD demand and probabilities of paths being chosen. The probabilities are calculated with a simple logit model in which the utility function is path travel time. In this research, we applied the simple logit model with the same configuration as Tang and Friedrich [39].

Link travel times are initialized by applying free flow travel time, and then travel time is differently estimated before and after signal timing plan design. Before signal timing plan is designed, as no signal timing information is available, the link costs are updated by BPR function (see (4)). After signal timing plan is designed, the link travel time is updated by the sum of free flow travel time and link delay (see (5)). Link delay is the function of signal timing. Since during the first few iterations of SUE some of the links can be oversaturated, Akcelik's formula [40] is used. In Akcelik's formula, the delay consists of uniform delay and incremental delay which are represented as the first and the second term of (6), respectively.

$$
\begin{aligned}
t_{a}\left(q_{a}\right) & =t_{0, a}\left(1+\alpha\left(\frac{q_{a}}{Q_{a}}\right)^{\beta}\right) \\
t_{a}\left(q_{a}, \eta\right) & =t_{0, a}+d_{a}\left(q_{a}, \eta\right),
\end{aligned}
$$

where $a \in M$ is link index; $t_{a}\left(q_{a}\right)$ is travel time of link $a$; $t_{0, a}$ is free flow travel time of link $a(\mathrm{~h}) ; Q_{a}$ is capacity of link $a$ which is assigned as saturation flows of link $a$ (veh/h); $\alpha, \beta$ are parameters in BPR function; $d_{a}\left(q_{a}, \eta\right)$ is delay of link $a$ (h).

If link $a$ is on lane $k$ of arm $i$ at intersection $z$, the link delay $d_{a}\left(q_{a}, \eta\right)$ is then equal to $d_{z, i, k}\left(q_{z, i, k}, \eta\right)$.

$$
\begin{aligned}
& d_{z, i, k}\left(q_{z, i, k}, \eta\right)=\frac{0.5 c\left(1-g_{z, i, k} / c\right)^{2}}{1-\min \left(1, x_{z, i, k}\right) g_{z, i, k} / c} \\
& +\min \left(1,\left\lfloor\frac{x_{z, i, k}}{x_{0, z, i, k}}\right\rfloor\right) 900 T\left[\left(x_{z, i, k}-1\right)\right. \\
& +\sqrt{\left.\left(x_{z, i, k}-1\right)^{2}+\frac{12\left(x_{z, i, k}-x_{0, z, i, k}\right)}{Q_{z, i, k} T}\right]}
\end{aligned}
$$

where $k$ is lane index; $d_{z, i, k}\left(q_{z, i, k}, \eta\right)$ is delay of lane $k(\mathrm{~s}) ; q_{z, i, k}$ is total traffic flow in lane $k(\mathrm{veh} / \mathrm{h}) ; c$ is common cycle length $(\mathrm{s}) ; g_{z, i, k}$ is green duration of lane $k(\mathrm{~s}) ; Q_{z, i, k}$ is capacity of lane $k(\mathrm{veh} / \mathrm{h}) ; x_{z, i, k}=q_{z, i, k} / Q_{z, i, k}$ is degree of saturation flow of lane $k$; $T$ is observation time period (h); $x_{0, z, i, k}=0.67+$ $s_{z, i, k} g_{z, i, k} / 600$ is turning point where the delay dramatically increases; $s_{z, i, k}$ is saturation flow of lane $k(\mathrm{veh} / \mathrm{s})$.

2.4. Signal Timing Plan Design. This section first introduces the method of conflict matrix determination according to left turn phasing types. With the conflict matrix, the stages can be generated and their sequences can be optimized. To estimate the delays, one should also calculate the lane flow and adjust the saturation flow. Finally the signal timing is calculated, especially for the stages sharing movements.

2.4.1. Determination of Conflict Matrix for Left Turns. Conflict matrix records conflicts between movements which cannot be in the same signal stage for safety reasons. If left 
turns are protected, they will not be on the same stage as opposing through movements; if they are permitted, they can be on the same stage as opposing through movements. Thus, types of left turn phasing affect conflict matrix and saturation flow of left turns. As the permitted left turn vehicles are interrupted by opposing through movements, the saturation flows decrease compared with the saturation flows of protected left turns.

To determine whether left turns need to be protected, one can apply recommendations from different guidelines. However, most guidelines focus on safety aspects $[1,4]$. In our method, we apply the volume conditions. The volume condition refers to the left turn flows and the product of left turn flow and opposing through flow. Roess et al. [5] suggested that the left turn, whose flow is more than $200 \mathrm{veh} / \mathrm{h}$ and product is more than 50000, should be protected. HCM [41] set these conditions more specific, and they are applied in this paper. However, if a left turn has no opposing through movement, it is unnecessary to consider the types of left turn phasing. Any of the following conditions lead to protected left turns:

(i) $q_{\mathrm{LT}}>240$.

(ii) if $N_{L, \mathrm{TH}}=1, q_{\mathrm{LT}} q_{\mathrm{TH}}>50000$.

(iii) if $N_{L, \mathrm{TH}}=2, q_{\mathrm{LT}} q_{\mathrm{TH}}>90000$.

(iv) if $N_{L, \mathrm{TH}} \geq 3, q_{\mathrm{LT}} q_{\mathrm{TH}}>110000$.

Here $N_{L, \mathrm{TH}}$ is number of opposing through lanes; $q_{\mathrm{LT}}$ is left turn flow (veh/h); $q_{\mathrm{TH}}$ is opposing through flow (veh/h).

Please note that when a left turn is permitted according these conditions, the left turn could be either permitted or protected. In the conflict matrix, if two movements do not conflict, they still could be in the different stages. For the same reason, the permitted left turn and the opposing through movement could be in the different stage, so that the left turn is protected in this case, and when calculating the saturation flow of the left turn, one should regard the left turn as protected left turn. The final left turn phasing type is determined according to the generated stages.

2.4.2. Stage Generation. When generating the stages, one of the conventions is to include all nonconflict movements in the same stage (compatibility). In this regard, a feasible set would be one which contains different stages with nonconflict movements. It is equivalent to the problem of finding all subgraphs in graph theory. The adjacent matrix indicating the compatibility among movements is generated from the conflict matrix. If the value of the conflict matrix is 1 , two movements conflict; if the value is 0 , the movements are not in conflict. Respectively, if the value in the adjacent matrix is 1 , meaning that both movements are compatible, they can be in the same stage; if this value is 0 , the two movements are incompatible and therefore cannot be in the same stage, as shown in Figure 3. In some cases, the conflict matrix alone is not sufficient, since the lane configuration may generate additional incompatibility. For example, in Figure 3(a), lane 1 and lane 2 cannot share a stage with either lane 5 or lone 6 , because the left turn in lane 2 conflicts with both lanes 5 and 6 . However, once the adjacent matrix is generated, the compatibility of all moments can be extracted from there. As can be seen in Figure 3(b), in the conflict matrix, the through movement in arm 1 is not in conflict with the through moment in arm 3. Nevertheless, both of these movements are incompatible, as shown in the respective cell of the adjacent matrix (see Figure 3(c)).

Once we have all possible stages by finding all subgraphs (see Figure 3(d)), we should keep the number of stages as small as possible, to decrease the intergreen time. However, we need to make sure that all movements are included in our plan. To this end, we formulate the stage selection as a $0 / 1$ integer programming problem whose objective is to minimize the number of stages. The decision variables model whether a stage should be selected and the constraints represent the requirement that each movement must be included at least in one stage (completeness). Consequently, the minimum number of stages including all movements is generated. After the stages are generated, the left turn phasing types are fixed. Figure 3(e) illustrates an example of the stage generation. " $G$ " means green time and " $R$ " means red time. "LT" represents left turn, "TH" represents through movement, and "RT" represents right turn.

When the stages are generated, one movement may be shared by multiple stages. For example, in the numerical example of Memoli et al. [29], two stages share the through movement. This is rarely applied in the stage-based method. Sharing a movement by multiple stages influences the stage sequence and green duration of these stages. We further address the issue of stages sharing the same movements in Sections 2.4.3 and 2.4.7.

2.4.3. Stage Sequence Optimization. Generally the most favorable stage sequence is determined by the total necessary intergreen times, which leads to shortest cycle time [3]. In this paper, the stage sequence is optimized by minimizing the total intergreen time. From the adjacent matrix in the "stage generation" (see Figure 4(a)), the intergreen time matrix can be generated. In this matrix, the compatible movements have no intergreen time, whereas the incompatible movements do. The intergreen times between incompatible movements are mainly determined by the geometry of the intersection. The intergreen time between stages is determined based on the intergreen time matrix and the composition of the stages (see Figure 4(b)).

If one movement appears in multiple sequential stages, the signal of the movement can keep green in the intergreen time between stages; i.e., the intergreen time is 0 . The movement thereby has extra green time and capacity. However, if these stages are not sequential, the extra green time cannot be gained. Therefore, when determining the stage sequence, one should consider the potential gain of extra green time. In this paper, the stage sequence optimization problem is represented as the traveling salesman problem (TSP), where the sequence of stages is equivalent to the visited cities and the minimum total intergreen time is equivalent to the minimum distance (see Figure 4(e)). The distance between two stages is the sum of intergreen times among the related movements (see Figures 4(c) and 4(d)). In this regard, if the stages sharing the same movements are sequential, the distance between 


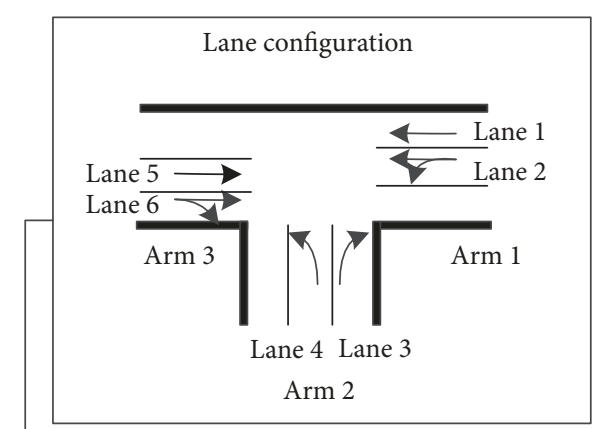

(a)

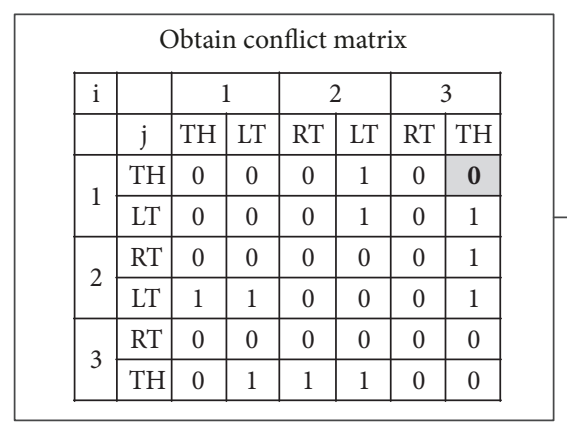

(b)

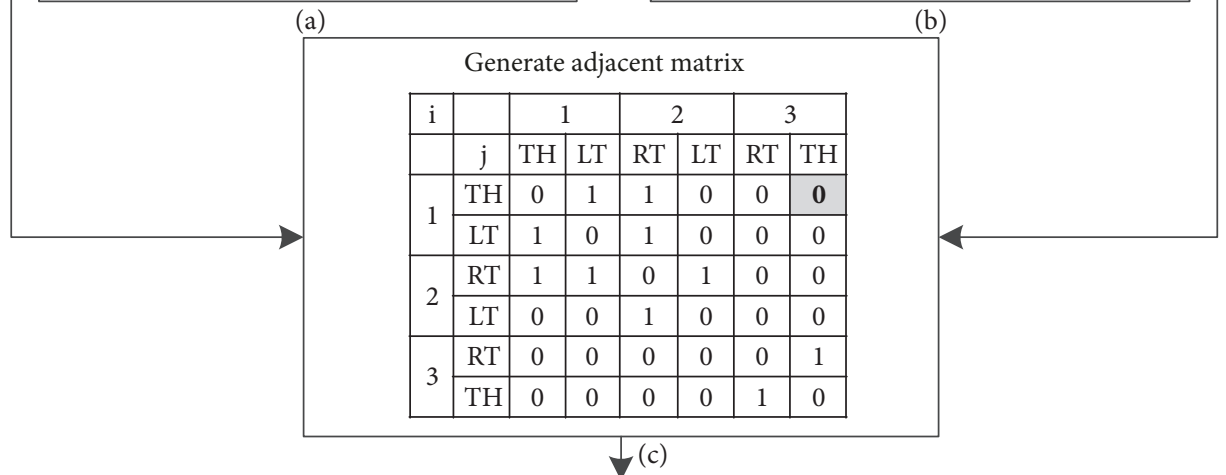

Generate feasible set by finding sub-graphs

\begin{tabular}{|c|c|c|}
\hline Arm 1 & Arm 2 & Arm 3 \\
\hline \begin{tabular}{l|l} 
TH & LT
\end{tabular} & \begin{tabular}{|l|l|} 
RT & LT \\
\end{tabular} & \begin{tabular}{l|l|} 
RT & TH \\
\end{tabular} \\
\hline
\end{tabular}

Stage 1 \begin{tabular}{|l|l|l|l|l|l|}
\hline G & G & G & R & R & R \\
\hline
\end{tabular}

Stage 2 \begin{tabular}{|l|l|l|l|l|l|}
\hline $\mathrm{R}$ & $\mathrm{R}$ & $\mathrm{G}$ & $\mathrm{G}$ & $\mathrm{R}$ & $\mathrm{R}$ \\
\hline
\end{tabular}

Stage 3 \begin{tabular}{|l|l|l|l|l|l|}
\hline R & R & R & R & G & G \\
\hline
\end{tabular}

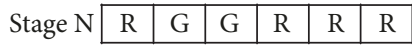

$\downarrow$ (d)

Select stages by minimizing the number of stages

\begin{tabular}{|c|c|c|}
\hline Arm 1 & Arm 2 & Arm 3 \\
\hline TH & LT & RT \\
\hline
\end{tabular}

\begin{tabular}{|l|l|l|l|l|l|}
\hline TH & LT & RT & LT & RT & TH \\
\hline
\end{tabular}

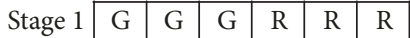

Stage 2 \begin{tabular}{|l|l|l|l|l|l|}
\hline R & R & R & R & G & G \\
\hline
\end{tabular}

\begin{tabular}{ll|l|l|l|l|l|} 
Stage 3 & $\mathrm{R}$ & $\mathrm{R}$ & $\mathrm{G}$ & $\mathrm{G}$ & $\mathrm{R}$ & $\mathrm{R}$ \\
\hline
\end{tabular}

(e)

FIGURE 3: An example of stage generation: (a) lane configuration; (b) obtaining conflict matrix; (c) generation of adjacent matrix; (d) generation of feasible set by finding subgraphs; (e) selecting stages by minimizing the number of stages.

each stage is shorter than the distance of nonsequential stages sharing the same movements.

Denote $i$ as the arm index and $N_{A, z}$ is the number of arms at intersection $z$. Denote movement index $j$ in the turning direction set $J=\{\mathrm{LT}, \mathrm{TH}, \mathrm{RT}\}$, where LT is left turn, and TH is through movement, and RT is right turn. Considering the types of left turn phasing, LT may also be denoted as permLT for permitted left turns and protLT for protected left turns. A movement is then defined as $(i, j)$ which indicates a turning direction $j$ from arm $i$.
Set formally, for intersection $z$, if a movement $(i, j)$ is in stage $p$ and a movement $(u, v)$ is in stage $p^{\prime}$, then the distance between both stages can be expressed as follows:

$$
l_{p, p^{\prime}}=\sum_{i=1}^{N_{A, z}} \sum_{j \in J} \sum_{u=1}^{N_{A, z}} \sum_{v \in J} l_{i, j, u, v},
$$

where $l_{p, p^{\prime}}$ is distance between stage $p$ and stage $p^{\prime} ; i, u$ denote arm index; $j, v$ denote turning direction index; $l_{i, j, u, v}$ denotes intergreen time between movements $(i, j)$ and $(u, v)$. 


\begin{tabular}{|c|c|c|c|c|c|c|c|c|c|c|c|c|c|}
\hline \multicolumn{14}{|c|}{ Obtain adjacent matrix } \\
\hline \multirow[t]{2}{*}{$\mathrm{i}$} & & \multicolumn{3}{|c|}{1} & \multicolumn{3}{|c|}{2} & \multicolumn{3}{|c|}{3} & \multicolumn{3}{|c|}{4} \\
\hline & $j$ & RT & $\mathrm{TH}$ & LT & RT & $\mathrm{TH}$ & LT & RT & $\mathrm{TH}$ & LT & RT & $\mathrm{TH}$ & LT \\
\hline \multirow{3}{*}{1} & RT & 0 & 1 & 1 & 0 & 0 & 0 & 0 & 0 & 0 & 1 & 1 & 1 \\
\hline & \begin{tabular}{|l|}
$\mathrm{TH}$ \\
\end{tabular} & 1 & 0 & 1 & \begin{tabular}{|l}
0 \\
\end{tabular} & \begin{tabular}{|l}
0 \\
\end{tabular} & 0 & 0 & 0 & 0 & 0 & 0 & 0 \\
\hline & \begin{tabular}{|l|} 
LT \\
\end{tabular} & 1 & 1 & 0 & 0 & 0 & 0 & 0 & 0 & 0 & 0 & 0 & 0 \\
\hline \multirow{3}{*}{2} & RT & 0 & 0 & 0 & 0 & 1 & 1 & 0 & 0 & 0 & 1 & 0 & 0 \\
\hline & $\mathrm{TH}$ & 0 & 0 & 0 & 1 & 0 & 1 & 0 & 0 & 0 & 0 & 0 & 0 \\
\hline & LT & 0 & 0 & 0 & 1 & 1 & 0 & 0 & 0 & 0 & 0 & 0 & 0 \\
\hline \multirow{3}{*}{3} & RT & 0 & 0 & 0 & 0 & 0 & 0 & 0 & 1 & 1 & 0 & 0 & 0 \\
\hline & $\mathrm{TH}$ & 0 & 0 & 0 & 0 & 0 & 0 & 1 & 0 & 1 & 0 & 0 & 0 \\
\hline & \begin{tabular}{|l|} 
LT \\
\end{tabular} & 0 & 0 & 0 & 0 & 0 & 0 & 1 & 1 & 0 & 0 & 0 & 0 \\
\hline \multirow{3}{*}{4} & RT & 1 & 0 & 0 & 1 & 0 & 0 & 0 & 0 & 0 & 0 & 1 & 1 \\
\hline & $\mathrm{TH}$ & 1 & 0 & 0 & 0 & 0 & 0 & 0 & 0 & 0 & 1 & 0 & 1 \\
\hline & LT & 1 & 0 & 0 & 0 & 0 & 0 & 0 & 0 & 0 & 1 & 1 & 0 \\
\hline
\end{tabular}

(a)

\begin{tabular}{|l}
\hline \multicolumn{10}{|c|}{ Obtain generated stages } \\
\begin{tabular}{|c|c|c|c|c|c|c|c|c|c|c|c|c|}
\hline \multicolumn{1}{|c|}{ Arm 1 } & \multicolumn{3}{c|}{ Arm 2 } & \multicolumn{3}{c|}{ Arm 3 } & \multicolumn{3}{c|}{ Arm 4 } \\
\hline RT & TH & LT & RT & TH & LT & RT & TH & LT & RT & TH & LT \\
\hline Stage 1 & G & G & G & R & R & R & R & R & R & R & R & R \\
Stage 2 & R & R & R & G & G & G & R & R & R & R & R & R \\
Stage 3 3 & G & R & R & R & R & R & R & R & R & G & G & G \\
\hline Stage 4 4 & R & R & R & R & R & R & G & G & G & R & R & R \\
\hline
\end{tabular}
\end{tabular}

(c)
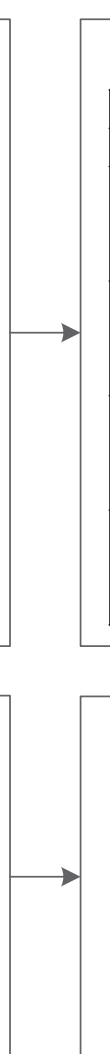

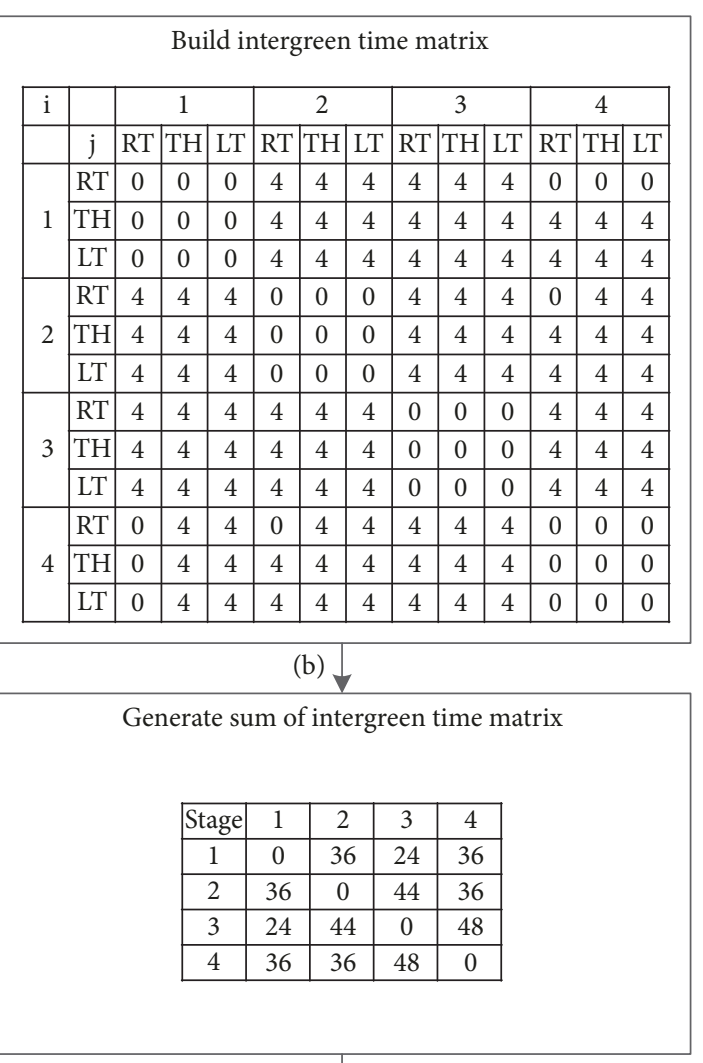

(d)

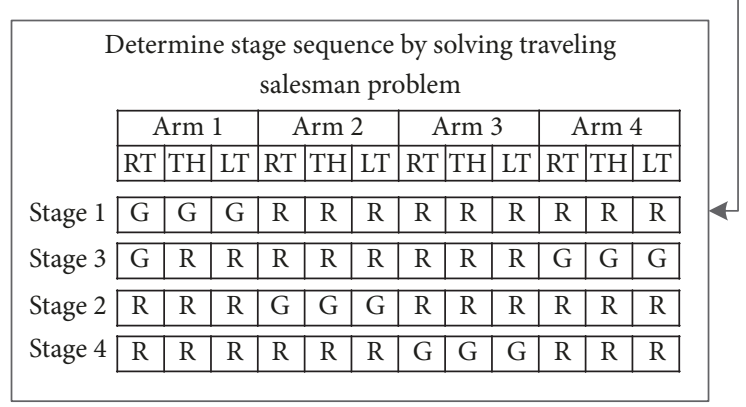

(e)

FIGURE 4: An example of stage sequence optimization: (a) obtaining adjacent matrix; (b) building intergreen time matrix; (c) obtaining generated stages; (d) generating sum of intergreen time matrix; (e) determining stage sequence by solving traveling salesman problem.

For example, in Figure 4, the intergreen times between each incompatible movements are $4 \mathrm{~s}$. As stages 1 and 2 do not share any movements, the distance between stages 1 and 2 is $36 \mathrm{~s}$. However, the right turn in arm 1 is shared by both stages 1 and 3, so the distance between stages 1 and 3 sums up to $24 \mathrm{~s}$. The signal for the right turn keeps green, so the intergreen time is gained. By applying TSP, stages 1 and 3 are sequential.

2.4.4. Lane Flow Calculation. As one movement may occupy multiple lanes, drivers have to select one lane for turning preparation. To well adjust signal timing, it is necessary to calculate the number of vehicles turning a direction on each lane, i.e., the assigned flow. Lane flow is the sum of assigned flows of each movement.
Denotations $k$ and $N_{L, i}$ are the lane index and the number of lanes on $\operatorname{arm} i$, respectively. For all intersection $z=$ $1, \ldots, N_{Z}$, arm $i=1, \ldots, N_{A, z}$, and turning direction $j \in J$, demands $F_{i, j}$ from each iteration of SUE with signal timing are equal to the sum of assigned flows of movement $(i, j)$.

$$
F_{i, j}=\sum_{k=1}^{N_{L, i}} q_{i, j, k}, \quad \forall i=1, \ldots, N_{A, z} ; j \in J,
$$

where $q_{i, j, k}$ is the assigned flow of movement $(i, j)$ on lane $k$.

If a movement $(i, j)$ is not on lane $k$, the assigned flow $q_{i, j, k}$ should be 0 .

$$
q_{i, j, k}=0, \quad \forall i=1, \ldots, N_{A, z} ; j \in J ; k=1, \ldots, N_{L, i}
$$


If movement $(i, j)$ is on multiple lanes, the saturation flow rates of all lanes tend to be identical because drivers enter the lane with the shortest queue, resulting in the even distribution of assigned flows [26].

$$
\begin{aligned}
\sum_{j \in J} \frac{q_{i, j, k}}{s_{j, k}}- & \sum_{j \in J} \frac{q_{i, j, k+1}}{s_{j, k+1}}=0, \\
& \forall i=1, \ldots, N_{A, z} ; j \in J ; k=1, \ldots, N_{L, i}-1,
\end{aligned}
$$

where $s_{j, k}$ is the saturation flow of movements on exclusive lanes.

By solving linear equations in (8)-(10), the assigned flows are determined. Specifically, if an assigned flow is less than 0 , the related movement cannot be assigned to that lane. Instead, the assigned flow is assigned to its adjacent feasible lanes until all assigned flows are nonnegative.

Consequently, the lane flow can be calculated via summing the assigned flow of each movement on the lane:

$$
q_{i, k}=\sum_{j \in M} q_{i, j, k}, \quad \forall i=1, \ldots, N_{A, z} ; k=1, \ldots, N_{L, i}
$$

2.4.5. Saturation Flow Adjustment. In Figure 2, after "determining types of left turn phasing" and before "calculating lane flows", the saturation flow of permitted/protected left turns can be adjusted. The saturation flow is adjusted once again, this time for shared lanes, after the "calculating lane flows" step.

The saturation flow of permitted left turns per lane is adjusted by estimating the filtered saturation flow based on gap acceptance theory and adjusting the saturation flow of an exclusive lane [40].

$$
\begin{gathered}
s_{\text {filtered }}=\frac{q_{\mathrm{TH}} \exp \left(-q_{\mathrm{TH}} l_{c}\right)}{1-\exp \left(-q_{\mathrm{TH}} l_{f}\right)} \\
s_{\text {permLT,k }}=s_{\mathrm{TH}} \frac{s_{\text {filtered }} g_{u}+N_{f}}{0.5 g_{\mathrm{LT}}},
\end{gathered}
$$

where $s_{\text {filtered }}$ is filtered saturation flow of permitted left turns (veh/s); $q_{\mathrm{TH}}$ is opposing through movement flow (veh/s); $l_{c}$ is critical gap with a value $4.5 \mathrm{~s} ; l_{f}$ is follow-up headway with value $2.5 \mathrm{~s} ; s_{\text {permLT }, k}$ is saturation flow of permitted left turns per lane (veh/s); $s_{\mathrm{TH}}$ is saturation flow of through movement per lane (veh/s); $g_{\mathrm{LT}}$ is green duration of left turns $(\mathrm{s}) ; g_{u}=$ $\max \left(\left(s_{\mathrm{TH}} g_{\mathrm{LT}}-q_{\mathrm{TH}} c\right) /\left(s_{\mathrm{TH}}-q_{\mathrm{TH}}\right), 0\right)$ is unsaturated part of the green period for the opposing through movement (s); $c$ is cycle length (s); $N_{f}$ is the number of vehicles passing during the amber period and is predefined as 1.5 (veh) in Akcelik (1981) and HCM (2000) unless field data are available.

The saturation flow of movements on the shared lane is weighted according to the flow of each movement $[26,40]$.

$$
s_{i, k}=\frac{1}{\sum_{j \in J} r_{j} / s_{j, k}},
$$

where $k$ is lane index; $r_{j}$ is ratio of lane flow $q_{i, j, k}$ to the total flow on lane $k ; s_{j}$ is saturation flow of turning direction $j$ (veh/h).
2.4.6. Signal Timing. The formulas of signal timing calculation are originally deduced by Webster [25]. HBS [26] and RiLSA [42] adopt these formulas in the manual. Webster's formulas can only be applied in unsaturated conditions which are regarded as the limitation. However, considering the constraints of cycle length, cycle length can be the maximum value if the degree of saturation is more than 1. Thus, Webster's formulas are feasible and applicable at the saturated intersections. Further, by selecting the largest cycle length of the network as the common cycle length, Webster's formulas can be also used in the network [27].

To calculate cycle length, the flow ratio, which is the flow to the saturation flow, for each stage must be determined. As green times are determined by the lane with maximum flow in a stage, for all lanes in the same stage, the maximum flow ratio of lanes is the flow ratio of the stage (see (14)).

For all $i, k$ related to stage $p$,

$$
\begin{aligned}
b_{z, p} & =\max \left(\frac{q_{z, i, k}}{s_{z, i, k}}\right) \\
c_{z} & =\frac{1.5\left(\sum_{p=1}^{N_{P, z}} l_{p}+5\right)}{1-B_{z}} \\
g_{z, p} & =\frac{b_{z, p}}{B_{z}}\left(c_{z}-\sum_{p=1}^{N_{P, z}} l_{p}\right)
\end{aligned}
$$

subject to $c_{z} \in\left[c_{\min }, c_{\max }\right], g_{p} \in\left[g_{\min , p}, c_{z}\right]$, where $p=$ $1, \ldots, N_{P, z}$ is stage index; $l_{p}$ is intergreen time of stage $p(\mathrm{~s})$; $B_{z}=\sum_{p=1}^{N_{P, z}} b_{p}$ is sum of flow ratios at intersection $z ; N_{P, z}$ is the number of phases at intersection $z ; c_{z}$ is cycle length of intersection $z ; c_{\min }$ is minimum cycle time $(\mathrm{s}) ; c_{\max }$ is maximum cycle time $(s) ; g_{\min , p}$ is minimum green duration of stage $p$ (s).

Cycle length must be greater than or equal to the minimum cycle length and not larger than the maximum cycle length. Thus, if $B_{z}>1-1.5\left(\sum_{p=1}^{N_{P, z}} l_{p}+5\right) / c_{\max }$, the cycle length is always maximum.

Due to the constraints of minimum green times, if the green time of stage $p$ is less than the minimum green time, the green time is equal to the minimum green time. These minimum green times are treated as the components of intergreen time. Because the value of the green time is fixed to the minimum, the green times can only be assigned for the rest of stages. The cycle time should be recalculated as the actual sum of lost time increases. The rest of effective green time has to be accommodated according to the stage flows.

$$
\begin{gathered}
c_{z}=\frac{1.5\left(\sum_{p=1}^{N_{p, z}} l_{p}+\sum_{p=1}^{N_{p^{\prime}, z}} g_{\min , p}+5\right)}{1-B_{z}^{\prime}} \\
g_{z, p}=\frac{b_{z, p}}{B^{\prime}}\left(c_{z}-\sum_{p=1}^{N_{P, z}} l_{p}-\sum_{p=1}^{N_{p^{\prime}, z}} g_{\min , p}\right)
\end{gathered}
$$

subject to $c_{z} \in\left[c_{\min }, c_{\max }\right], g_{p} \in\left[g_{\min , p}, c_{z}\right]$, where $N_{P^{\prime}, z}$ is the number of stages with minimum green time $P^{\prime}$ and $B_{z}^{\prime}=$ $B_{z}-\sum_{p=1}^{N_{p^{\prime}, z}} b_{z, p}$. 


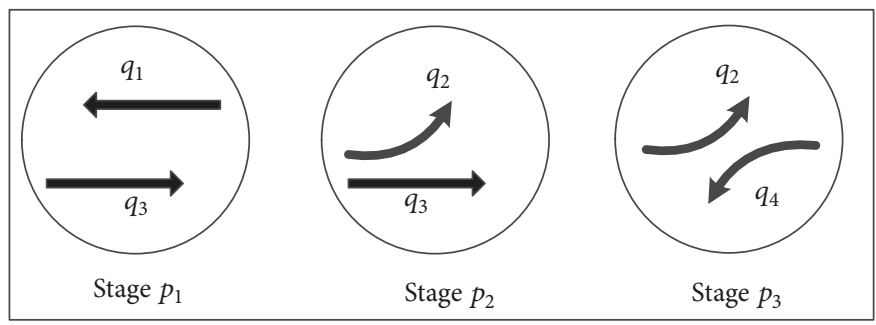

(a)

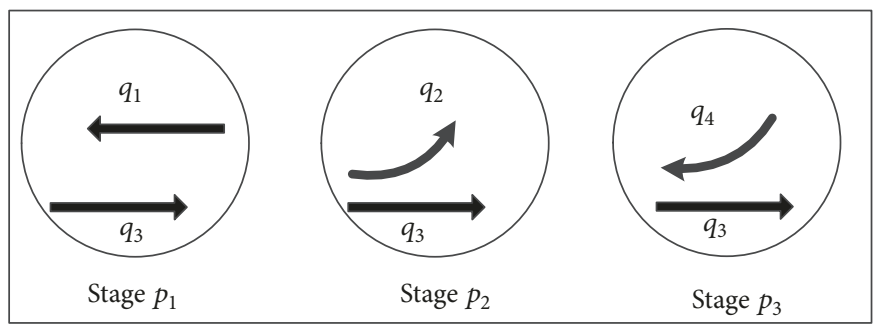

(b)

FIGURE 5: Examples of three stages sharing movements.

Cycle lengths are firstly calculated for each intersection, and the largest cycle length is decisive as the common cycle length for the network. Green times are then recalculated with the common cycle length by (16) and (18).

$$
c=\max c_{z}
$$

2.4.7. Signal Timing of Stages Sharing Movements. As mentioned, to calculate signal timing, the flows of movements/lanes in each stage should be known. However, if one movement is in two stages, it is hard to separate the movement flows into two stages beforehand and one cannot calculate the flow ratio. This case is neglected by HBS [26] and RiLSA [42] but considered by Pohlmann [38].

The rule to deal with the stages sharing movements is that green times are determined by the lane with the maximum flow. When calculating the green time of stages sharing movements, two cases need to be considered: either the required green time for the movement shared in multiple stages is bigger than the required green time for the other movements included in these stages or this green time is smaller. The final green time will be the larger one of these two cases. For example, in Figure 1, we assume each movement occupies one lane. For the movement with the flow $q_{3}$, we cannot know in advance the number of vehicles in stages $p_{1}$ and $p_{2}$, respectively. Hence, following HBS [26] recommendations, one cannot solve this case. As the largest flow determines the flow ratio in a stage, Pohlmann [38] separately calculated the flow ratio for stages $p_{1}$ and $p_{2}$ and combined stage $p_{1} p_{2}$. Stages only include the movement not shared by two stages, and the combined stage only include the movement shared by two stages. In Figure $1, b_{z, p_{1}}=q_{1} / s_{1}$ and $b_{z, p_{2}}=q_{2} / s_{2}$, but $b_{z, p_{1} p_{2}}=q_{3} / s_{3}$, and then the flow ratio of stages $p_{1}$ and $p_{2}$ is determined by $\max \left(b_{z, p_{1}}+b_{z, p_{2}}, b_{z, p_{1} p_{2}}\right)$.
With the same logic, the green time of stage $p_{1}$ and $p_{2}$ is determined by $\max \left(g_{z, p_{1}}+g_{z, p_{2}}, g_{z, p_{1} p_{2}}\right)$.

$$
g_{z, p_{1} p_{2}}=\frac{b_{z, p_{1} p_{2}}}{B_{z}^{\prime}}\left(c_{z}-\sum_{p=1}^{N_{P, z}} l_{p}-\sum_{p=1}^{N_{p^{\prime}, z}} g_{\min , p}\right)
$$

If $g_{z, p_{1} p_{2}}>g_{z, p_{1}}+g_{z, p_{2}}$,

$$
\begin{aligned}
& g_{z, p_{1}}=\frac{b_{z, p_{1}}}{b_{z, p_{1}}+b_{z, p_{2}}} g_{z, p_{1} p_{2}} \in\left[g_{\min , p_{1}}, c_{z}\right] \\
& g_{z, p_{2}}=g_{z, p_{1} p_{2}}-g_{z, p_{1}}
\end{aligned}
$$

else $g_{z, p_{1}}$ and $g_{z, p_{2}}$ do not change.

Pohlmann [38] considered only two stages sharing movements. Although this is usually the case, there may also be cases including more than two stages. Generalizing Pohlmann's method [38] to include more than two stages is not straightforward. For example, taking into account three stages sharing movements, the following process should be performed. First, we should consider the fact that not all three stages necessarily share the same movements, and movements may be shared only by two stages, as demonstrated in Figure 5(a). There, stages $p_{1}$ and $p_{2}$ share the through movement, and stages $p_{2}$ and $p_{3}$ share the left turn. Therefore, in order to calculate the green time, we should calculate $\max \left(b_{z, p_{1}}+b_{z, p_{2}}+b_{z, p_{3}}, b_{z, p_{1}}+b_{z, p_{2} p_{3}}, b_{z, p_{1} p_{2}}+b_{z, p_{3}}\right)$. A different calculation should be carried out for the case presented in Figure 5(b). There, the through movement is shared by all three stages, and therefore the flow ratio would be $\max \left(b_{z, p_{1}}+\right.$ $\left.b_{z, p_{2}}+b_{z, p_{3}}, b_{z, p_{1} p_{2} p_{3}}\right)$. The green duration is calculated in a similar manner.

\section{Algorithms}

In "prohibiting left turn" of Figure 2, the left turns are selected by the genetic algorithm with the default configuration of Java 


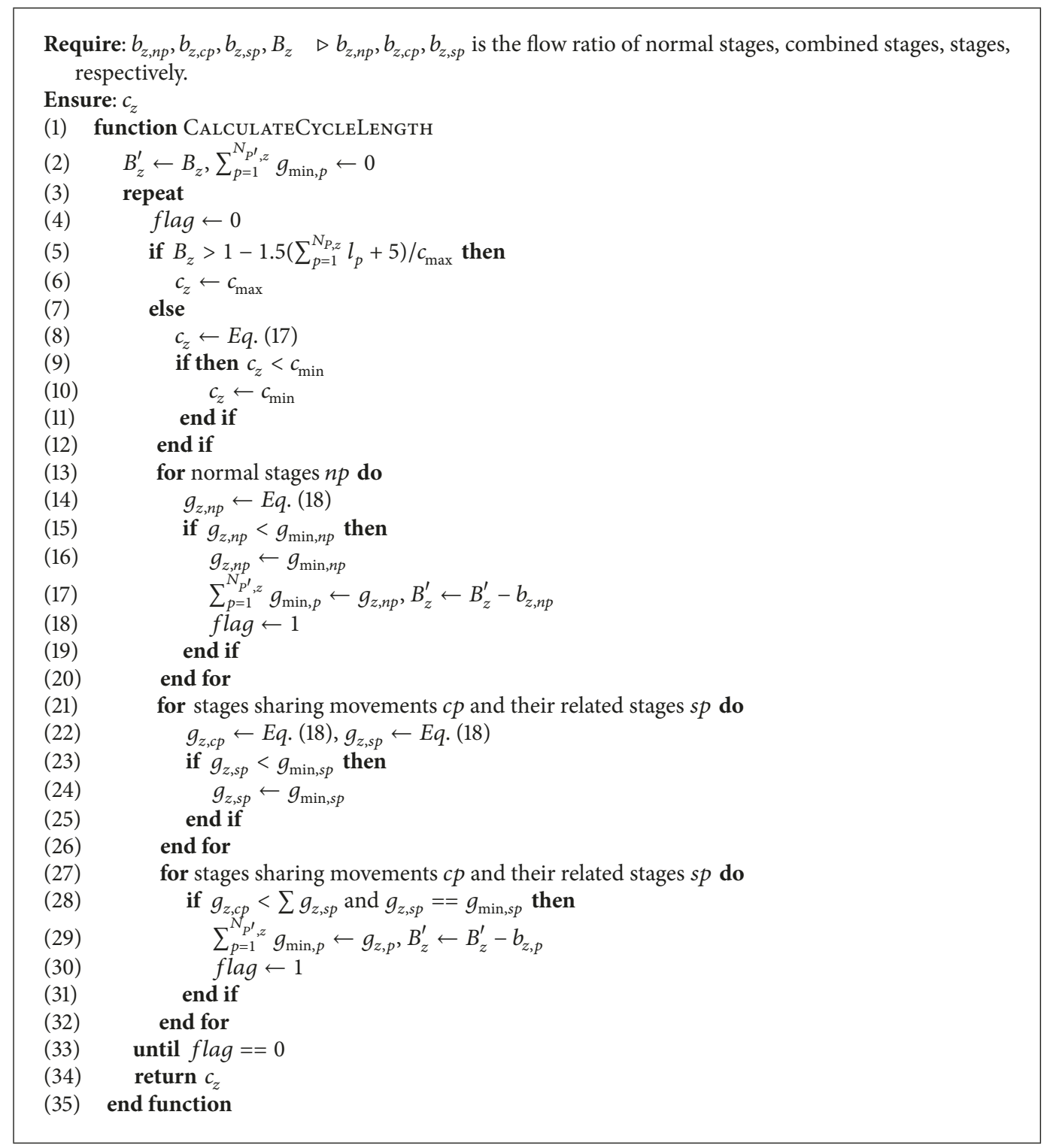

Algorithm 1: Calculation of cycle length for intersection $z$.

genetic algorithm package (Jgap) [43] which refers to an elitist ranking selector cloning the top $90 \%$ of the user-specified population size with crossover rate $35 \%$ and mutation rate $1 / 12$.

SUE is solved with the Method of Successive Average [44]. The link travel times are initialized with the free flow travel times of the links and then the initial link flows are estimated with stochastic loading based on the free flow travel times. In each iteration, the link travel times are updated with the link flows from the previous iteration, and the link flows are updated with the adjustment on the difference between the link flows from stochastic loading and the link flows from the previous iteration. The interaction between the link flows and the link travel times repeats until the convergence criteria are met.

For signal-relevant steps in Figure 2, "generating stages" is solved by finding all subgraphs with depth-first-search algorithm and minimization of the number of stages with routine branch and cut algorithm in IBM Ilog Cplex. The traveling salesman problem in stage sequence is determined by applying backtracking algorithm. The focus of this section is to explain signal timing calculation in the case of combined stages which is extended from [38]. The cycle length for each intersection is firstly calculated in Algorithm 1 and then the largest cycle length is selected as the common cycle length with which the green duration for each stage is calculated in Algorithm 2.

\section{Numerical Analysis}

4.1. Test Networks. The test network is an artificial network with six signalized intersections, ten origins, and ten destinations (Figure 6). There are 24 left turns. The free flow speed of each link is $50 \mathrm{~km} / \mathrm{h}$, and each link has three lanes. 


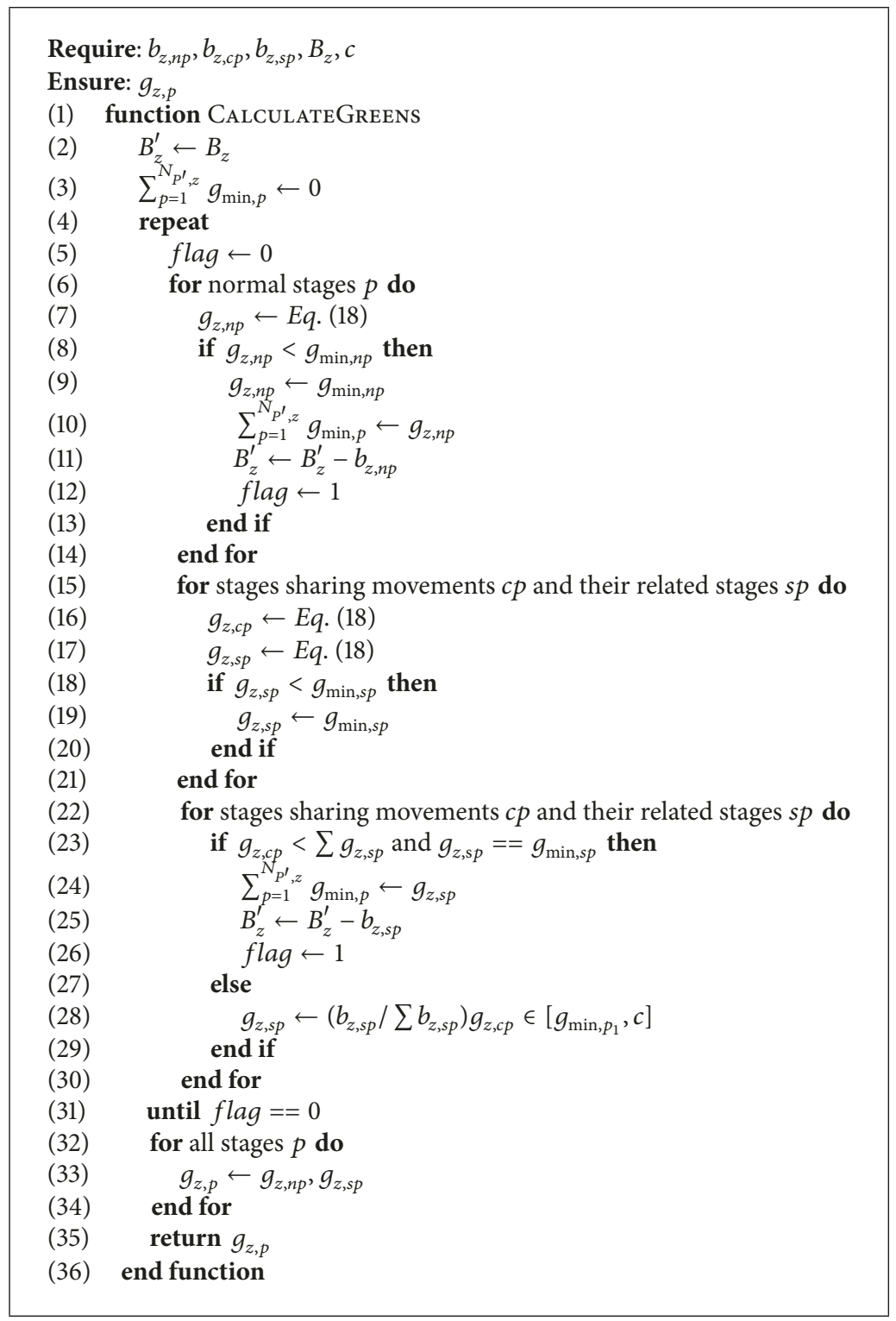

Algorithm 2: Calculation of green duration for each stage.

The default conflict matrices are extracted from network files of SUMO, which is a microscopic simulation tool developed by the German Aerospace Center (DLR). The network is tested with the OD matrix in Table 1 . The values of parameters are in Table 2. Specifically, the intergreen times are assumed to independent of the intersection geometry and the intergreen times between all stages are constant. In SUE, the convergence criterion is that the difference of average link flows between adjacent iterations is less than $1.0 \times 10^{-4}$. In the genetic algorithm, the initial population size is 50 , and the generation size is 30 .

4.2. Results. With the OD matrix and test network, 3 of 24 left turns are prohibited: the left turn in the northern arm at Intersection 2, the left turn in the southern arm at Intersection 3, and the left turn in the western arm at
Intersection 6. Before LTP, the total travel time is $564.9 \mathrm{~h}$ whereas, after LTP, the minimal total travel time is $435.5 \mathrm{~h}$ with reduction of $22.9 \%$. Prohibiting left turns reduces the total travel time. The average degrees of saturation for each intersection before and after LTP are also compared (see Figure 7). Except Intersection 4, the average degrees of saturation of the intersections decrease, especially at the intersections with LTP.

Signal settings are also adjusted before and after LTP. The common cycle lengths before LTP and after LTP are $100 \mathrm{~s}$. The stage generation results and their sequences before and after LTP are shown in Figure 8. The green durations before and after LTP are given in Table 3. After LTP, the number of stages at Intersection 6 becomes smaller which indicates a less intergreen time and longer effective green time. 
TABLE 1: Demands between origins and destinations (unit: veh/h).

\begin{tabular}{lccccccccccc}
\hline Origin/Destination & $\mathrm{A}$ & $\mathrm{B}$ & $\mathrm{C}$ & $\mathrm{D}$ & $\mathrm{E}$ & $\mathrm{F}$ & $\mathrm{G}$ & $\mathrm{H}$ & $\mathrm{I}$ & $\mathrm{J}$ & Total \\
\hline $\mathrm{A}$ & 0 & 70 & 90 & 160 & 100 & 60 & 150 & 50 & 180 & 200 & 1060 \\
B & 200 & 0 & 130 & 60 & 140 & 70 & 90 & 130 & 80 & 110 & 1010 \\
C & 180 & 90 & 0 & 180 & 90 & 160 & 70 & 80 & 120 & 70 & 1040 \\
D & 40 & 70 & 140 & 0 & 80 & 120 & 60 & 50 & 150 & 80 & 790 \\
E & 160 & 130 & 70 & 140 & 0 & 90 & 140 & 150 & 50 & 50 & 980 \\
F & 90 & 80 & 110 & 80 & 170 & 0 & 50 & 100 & 120 & 50 & 850 \\
G & 60 & 70 & 200 & 150 & 80 & 80 & 0 & 90 & 140 & 100 & 970 \\
H & 90 & 80 & 40 & 110 & 200 & 70 & 160 & 0 & 80 & 120 \\
I & 60 & 80 & 100 & 120 & 50 & 100 & 90 & 200 & 0 & 950 \\
J & 90 & 180 & 40 & 70 & 0 & 120 & 60 & 90 & 100 & 0 \\
Total & 970 & 850 & 920 & 1070 & 910 & 870 & 870 & 940 & 1020 & 870 & 950 \\
\hline
\end{tabular}

TABLE 2: Values of parameters.

\begin{tabular}{|c|c|c|}
\hline Parameters & Notations & Values \\
\hline Parameter in BPR function & $\alpha$ & 0.15 \\
\hline Parameter in BPR function & $\beta$ & 4 \\
\hline Number of exit lanes & $N_{\epsilon, z}, \forall z=1, \ldots, N_{Z}$ & 3 \\
\hline Saturation flow of through movements & $s_{\mathrm{TH}, k}, \forall k=1, \ldots, N_{L, i}$ & $1900 \mathrm{veh} / \mathrm{h}$ \\
\hline Saturation flow of right turns & $s_{\mathrm{RT}, k}, \forall k=1, \ldots, N_{L, i}$ & $1615 \mathrm{veh} / \mathrm{h}$ \\
\hline Saturation flow of protected left turns & $s_{\text {protLT, } k}, \forall k=1, \ldots, N_{L, i}$ & $1805 \mathrm{veh} / \mathrm{h}$ \\
\hline Saturation flow of permitted left turns & $s_{\text {permLT }, k}, \forall k=1, \ldots, N_{L, i}$ & by Eq. (12) \\
\hline Maximum cycle length & $c_{\max }$ & $60 s$ \\
\hline Minimum cycle length & $c_{\min }$ & $100 \mathrm{~s}$ \\
\hline Minimum green duration & $g_{\min , p}, \forall p=1, \ldots, N_{P, z}$ & $6 s$ \\
\hline Intergreen time & $l_{p}, \forall p=1, \ldots, N_{P, z}$ & $4 \mathrm{~s}$ \\
\hline Observation time & $T$ & $0.25 \mathrm{~h}$ \\
\hline
\end{tabular}

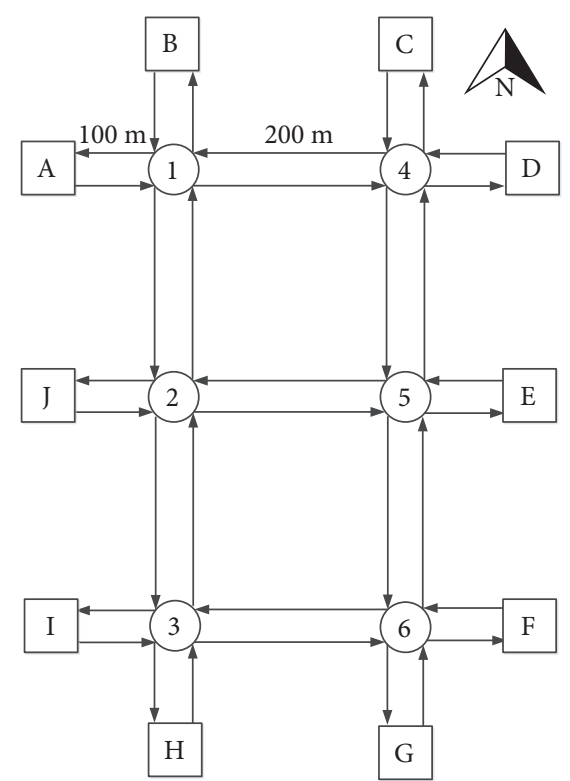

FIGURE 6: Layout of "toy" network with six intersections.

4.3. Evaluation of Algorithms. The algorithms of the proposed model are evaluated with increasing scale of networks.

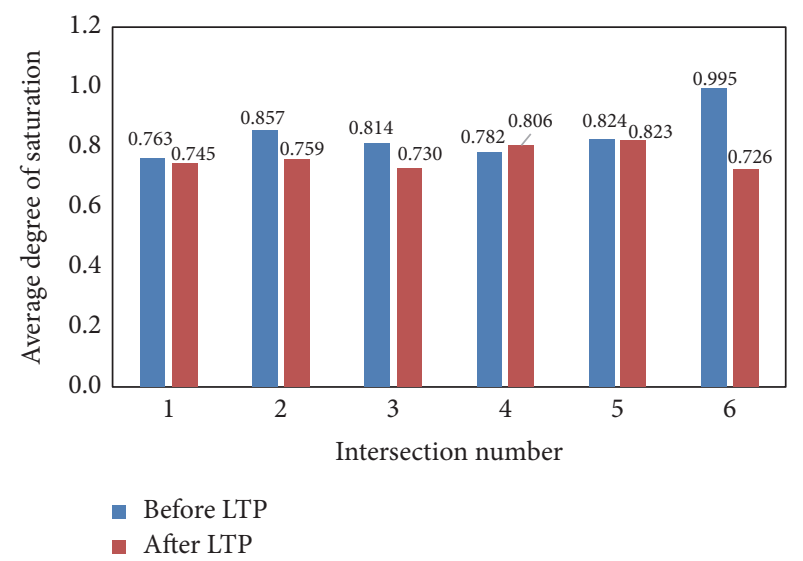

FIGURE 7: Average degree of saturation for each intersection before and after LTP.

Table 4 shows the information of the increasing network scales. The number of origins and the number of destinations are fixed to ten, which is the same as the original network (Network 1). For Network 2 to Network 7, the number of intersections increases one by one, resulting in the increasing number of nodes and links. The experiments have been 
(1)

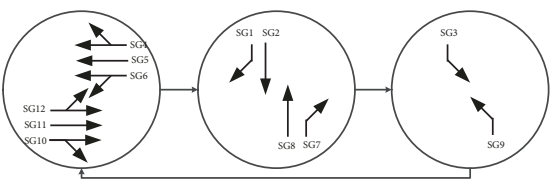

(2)

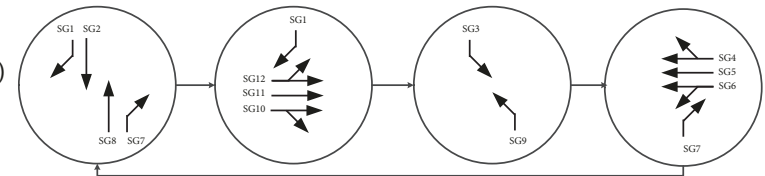

(3)

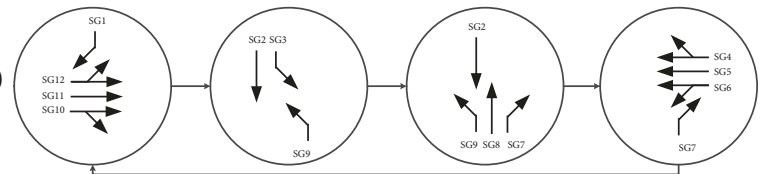

(4)

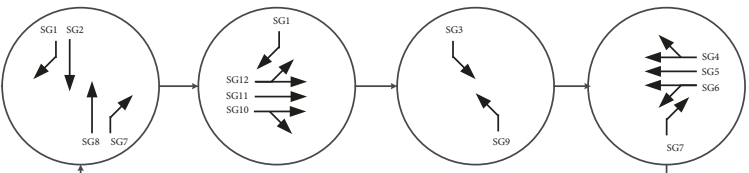

(5)

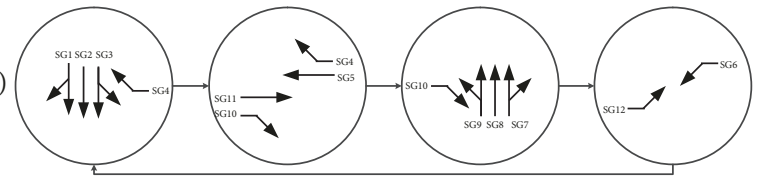

(6)

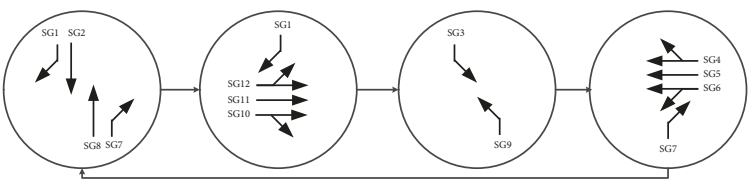

(a)
(1)

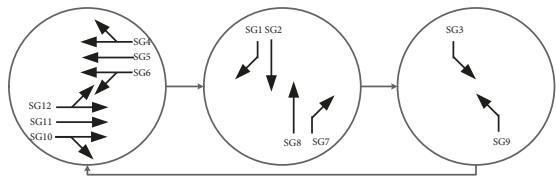

(2)

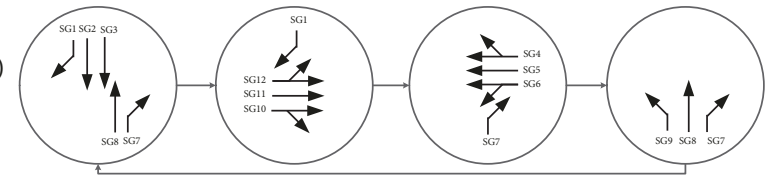

(3)

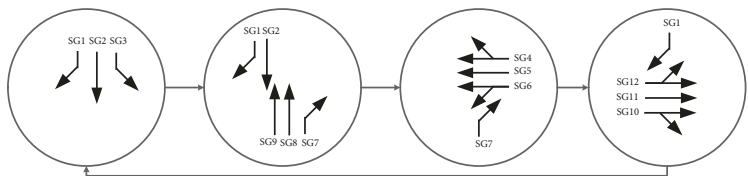

(4)

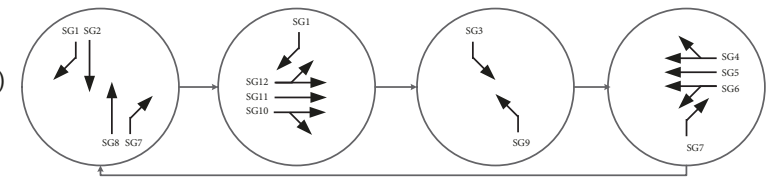

(5)

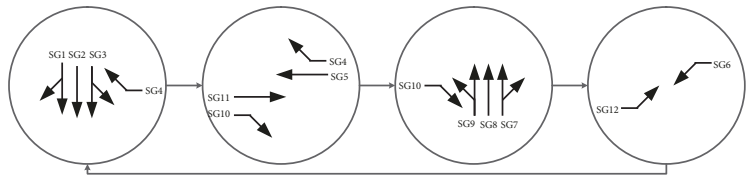

(6)

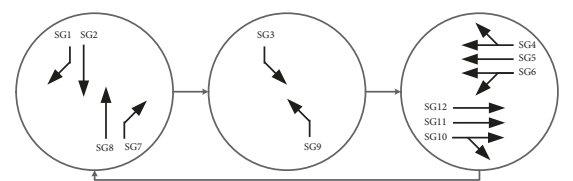

(b)

FIGURE 8: (a) Generated stages of test network and their sequence before LTP; (b) generated stages of test network and their sequence after LTP.

TABLE 3: Green durations of stages before and after LTP (s).

(a) Green durations of movements before LTP

\begin{tabular}{lcccc}
\hline Intersection & Stage 1 & Stage 2 & Stage 3 & Stage 4 \\
\hline 1 & 38 & 27 & 23 & \\
2 & 34 & 22 & 14 & 14 \\
3 & 33 & 11 & 24 & 16 \\
4 & 27 & 18 & 12 & 27 \\
5 & 23 & 16 & 26 & 19 \\
6 & 22 & 17 & 22 & 23 \\
\hline
\end{tabular}

(b) Green durations of movements after LTP

\begin{tabular}{lcccc}
\hline Intersection & Stage 1 & Stage 2 & Stage 3 & Stage 4 \\
\hline 1 & 35 & 28 & 25 & \\
2 & 22 & 18 & 22 & 22 \\
3 & 15 & 19 & 17 & 33 \\
4 & 26 & 21 & 11 & 26 \\
5 & 27 & 17 & 20 & 20 \\
6 & 30 & 27 & 31 & \\
\hline
\end{tabular}

conducted on an IPC with a $2.9 \mathrm{GHz}$ quad-core Intel Core i7 and with 16 GB memory. By applying these networks with the proposed model, the computing time for each network is illustrated in Figure 9. The computing time dramatically increases when the number of intersections is larger than 10. As routing search is important in the SUE algorithm, the network scale significantly influences the computing time. However, as the genetic algorithm may randomly select infeasible left turns, it immediately receives penalty and the total travel time is assigned to a constant large value, which means the SUE model and the design of signal timing plan are skipped. Thus, the computing time varies without an obvious tendency.

4.4. Analysis of Prohibited Left Turns. The flows of prohibited left turn and their types are analyzed. LTP is related to left turn flows according to previous research. Hajbabaie et al. [45] found that low LT volumes and oncoming high volumes led to LTP at some intersections. Pline [4] summarized that LT volume was a critical factor leading to prohibition. In consideration of a protected left turn for small flows wasting 
TABLE 4: Network scale information for algorithm evaluation.

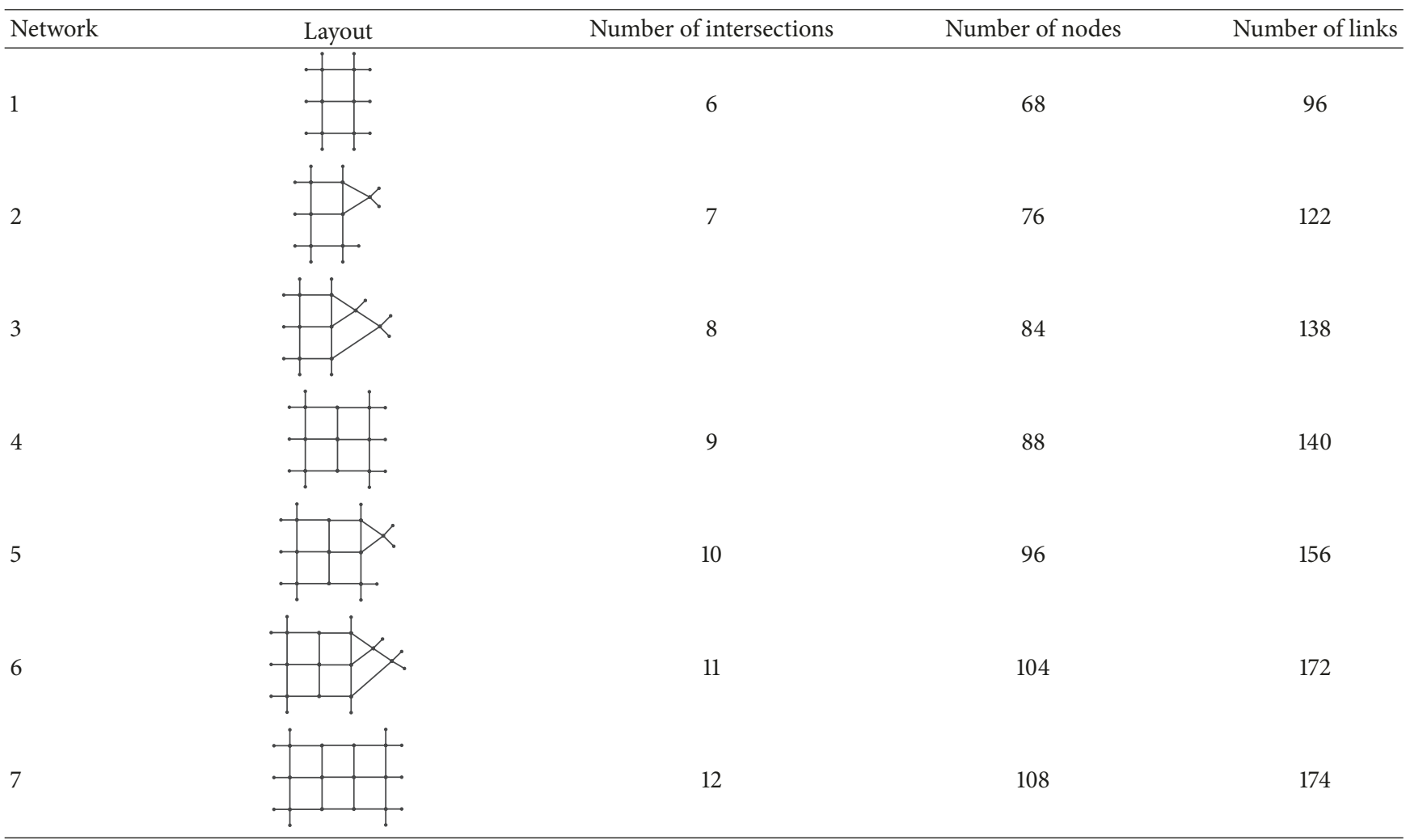

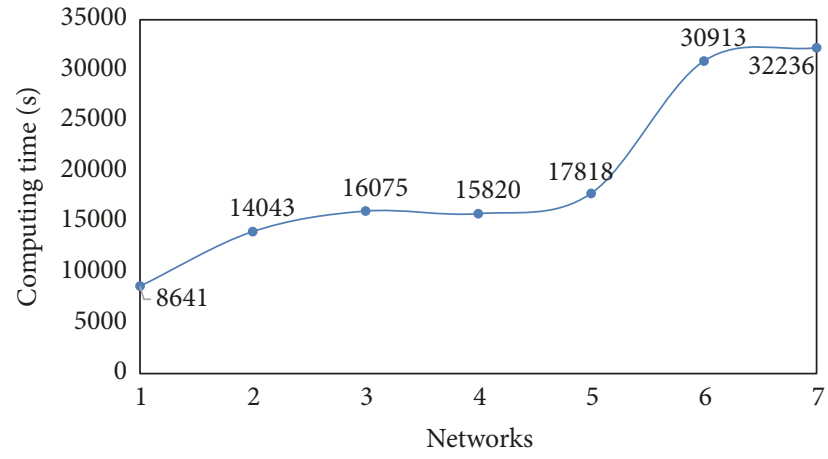

FIGURE 9: Computing time with increasing scale of networks.

capacity, we suppose that LTP is also related to the types of left turn phasing types. Permitted left turn flows justify conflict with the opposing straight vehicles, so whether permitted left turns should be prohibited depends on both left turn flows and opposing flows.

The flows and types of left turns without LTP and the LTP results from running the proposed model are collected via testing eight OD matrices. The OD matrices are generated by the demands in Table 1 plus random numbers in the ranges of $[-40,-30],[-30,-20],[-20,-10],[-10,0],[0,10],[10,20]$, $[20,30]$, and $[30,40]$, respectively. Including the original OD matrix, the flows of 214 left turns are collected and 36 of them are prohibited. We summarized the minimum, maximum, and the average value of all permitted left turns and protected left turns. We also calculated the average flow of all prohibited/permitted left turns and prohibited/permitted left turns.

The results are displayed in Figure 10. The green dots represent the average flows of permitted/protected left turns, and the high points of the vertical lines represent their maximum flow, and the low points of the vertical lines represent their minimum flow. The blue dots represent the average flows of prohibited/permitted/protected left turns. As no reduction from LTP can be found with the OD matrix having the random demands in the range of $[-10,0]$, no left turns are prohibited with this OD matrix. Further, as the demands increase, there are no permitted left turns for the OD matrices having random demands in the ranges of $[20,30]$ and $[30,40]$, respectively; and thus for these ranges no data is depicted in Figure 10(a). In Figure 10, all of the flows of the prohibited permitted left turns are smaller than the flows of all permitted left turns and the same as those for the protected left turns.

4.5. Discussion. The goal of this paper is to design signal timing plane including prohibiting left turns. The numerical example shows that prohibiting left turns reduces the total travel time. This study could potentially lead to useful insights regarding congestion management.

The main reason why prohibiting left turns can reduce the total travel time is that LTP reduces the number of conflict points at intersections. Due to the reduction of the conflict points, the number of stages decreases so that the intergreen 


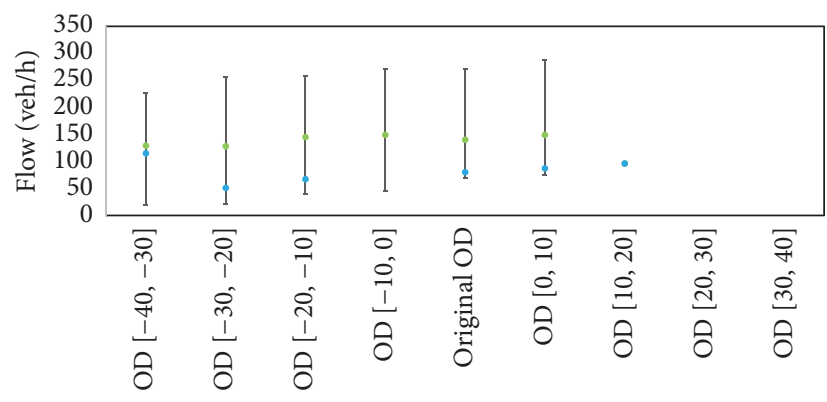

Permitted left turn

- Prohibited permitted left turn

(a)

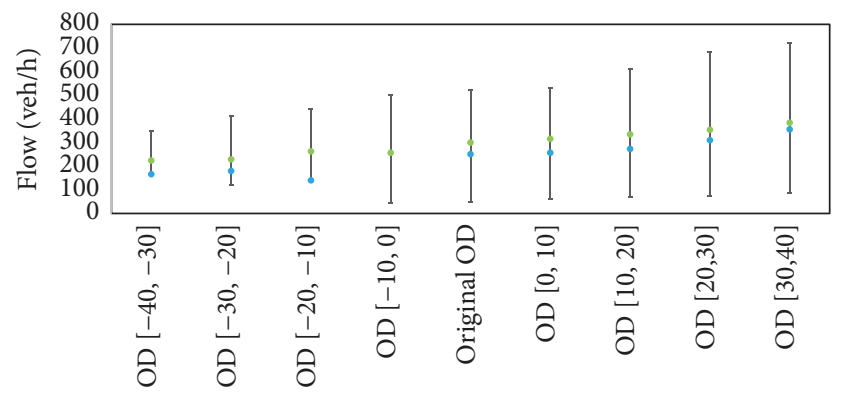

- Protected left turn

- Prohibited protected left turn

(b)

FIGURE 10: (a) Flow comparison between permitted left turns and prohibited/permitted left turns; (b) flow comparison between protected left turns and prohibited protected left turns.

time between two stages decreases (e.g., Intersection 6). If the intergreen time between two stages is longer, e.g., $6 \mathrm{~s}$, the total travel time reduction is expected to be larger because longer intergreen time indicates a high potential of more effective green time. Moreover, some movements are in multiple stages which also lengthens effective green times for those movements (e.g., the right turn in the northern arm of Intersection 3). Further, the lanes of prohibited left turns are assigned for through movements which also increases the capacity of the through movements (e.g., the through movement in the northern arm of Intersection 2). Therefore, the delays at the intersection go down and the total travel time decreases.

The prohibited left turns may be related to types of left turn phasing. The protected left turns with minor flows should be prohibited, but as the influence of opposing flows is not clear, we cannot conclude that the permitted left turns with minor flows should also be prohibited. Meanwhile, these findings are specific for the network. More generalized findings would require an additional analysis.

The signal timing plan with the proposed method does not aim at reaching global optimum. The design of signal timing consists of "stage generation", "stage sequence optimization", and "signal timing determination". Although "stage generation" and "stage sequence optimization" are conducted with optimization methods, "signal timing determination" is based on the formulas from the relevant manuals. The accumulation of the reasonable steps does not ensure the global optimization. Meanwhile, the interaction between signal timing and traffic flow is conducted in a straightforward way: with given traffic flows obtained from an SUE with a cost based on BRP function, a signal timing plan is designed for each intersection, and then the traffic flows adopt to the signal timing plan. The multiple interactions are not considered as the case in the dynamical scenarios.

\section{Conclusions}

We propose a method of designing signal timing plan including LTP by minimizing total travel time. The total travel time reduces after LTP. Types of left turn phasing and relevant left turn flows may be related to LTP. This paper provides an idea of congestion management in urban road networks. Prohibiting left turns should actually be considered among other left turn treatments in signal timing plan design. Planned future research includes analyzing the other factors influencing LTP.

\section{Data Availability}

The data used to support the findings of this study are available from the corresponding author upon request.

\section{Conflicts of Interest}

The authors declare that they have no conflicts of interest.

\section{Acknowledgments}

This research is supported by the German Research Foundation (DFG) through the Research Training Group SocialCars (GRK 1931). The focus of the SocialCars Research Training Group is on significantly improving the city's future road traffic through cooperative approaches. This work is also carried out within the project OptimUM (Optimization of Urban Traffic Management towards Environment Friendly and Safe Mobility) which is financially supported by the Helmholtz CAS Joint Research Groups (HCJRG) of the Helmholtz Association of German Research Centers. The support is gratefully acknowledged.

\section{References}

[1] K. R. Agent, "Guidelines for the Use of Protected/permissive Left-turn Phasing," Technical Report No. UKTRP-85-19, Kentucky Transportation Research Program, College of Engineering, University of Kentucky, 1985.

[2] J. H. Kell and I. J. Fullerton, Manual of traffic signal design, Prentice Hall, 1991.

[3] RiLSA., "Guidelines for Traffic Signals RiLSA," Tech. Rep., Cologne, Germany, 1992. 
[4] J. L. Pline, "Left-Turn Treatments at Intersections," Number NCHRP Sytthesis of Highway Practice 225, Transportation Research Board, 1996.

[5] R. P. Roess, E. S. Prassas, and W. R. McShane, Traffic Engineering, Prentice Hall, 2004.

[6] ADOT. ADOT Traffic Engineering Guidelines and Processes: section 612. Technical Report, Arizona Department of Transportation, 2015.

[7] J. Lu, S. Dissanayake, H. Zhou, and X. K. Yang, "Operational evaluation of right turns followed by $\mathrm{u}$-turns as an alternative to direct left turns the report based on "Methodology to quantify the effects of access management on roadway operation and safety", III," Tech. Rep., 2001.

[8] J. G. Bared and E. I. Kaisar, "Median U-turn design as an alternative treatment for left turns at signalized intersections," ITE Journal (Institute of Transportation Engineers), vol. 72, no. 2, pp. 50-54, 2002.

[9] M. Chowdhury, N. Derov, and P. Tan, "Evaluating the effects of prohibiting left turns and the resulting U-turn movement," FHWA/OH-2003/001, University of Dayton,Ohio Department of Transportation, Federal Highway Administration, 2003.

[10] P. Liu, J. J. Lu, H. Zhou, and G. Sokolow, "Operational effects of U-turns as alternatives to direct left-turns," Journal of Transportation Engineering, vol. 133, no. 5, pp. 327-334, 2007.

[11] L. Junqiang, Z. Hantao, and Z. Qian, "Research on the impact of U-turn location on operation efficiency at intersection," in Proceedings of the 2009 International Conference on Measuring Technology and Mechatronics Automation, ICMTMA 2009, pp. 567-570, April 2009.

[12] J. Zhao, W. Ma, K. L. Head, and Y. Han, "Improving the operational performance of two-quadrant parclo interchanges with median U-turn concept," Transportmetrica B, pp. 1-21, 2016.

[13] J. A. Bonneson and P. T. McCoy, "Capacity and operational effects of midblock left-turn lanes," Technical Report NCHRP Rep. 395, Transportation Research Board, Washington, DC, USA, 1981.

[14] M. Chowdhury, N. Derov, P. Tan, and A. Sadek, "Prohibiting left-turn movements at mid-block unsignalized driveways: Simulation analysis," Journal of Transportation Engineering, vol. 131, no. 4, pp. 279-285, 2005.

[15] S. Gyawali, A New Decision Making Approach for Indirect Left Turn Treatments by Utilizing Decision Assistance Curves [Ph.D. thesis], 2014.

[16] J. Zhao, M. Yun, H. M. Zhang, and X. Yang, "Driving simulator evaluation of driversâ response to intersections with dynamic use of exit-lanes for left-turn," Accident Analysis \& Prevention, vol. 81, pp. 107-119, 2015.

[17] J. Zhao, Y. Liu, and T. Wang, "Increasing Signalized Intersection Capacity with Unconventional Use of Special Width Approach Lanes," Computer-Aided Civil and Infrastructure Engineering, vol. 31, no. 10, pp. 794-810, 2016.

[18] W. Ma, H. Xie, Y. Liu, L. Head, and Z. Luo, "Coordinated optimization of signal timings for intersection approach with presignals," Transportation Research Record, no. 2355, pp. 93104, 2013.

[19] Y. Xuan, C. F. Daganzo, and M. J. Cassidy, "Increasing the capacity of signalized intersections with separate left turn phases," Transportation Research Part B: Methodological, vol. 45, no. 5, pp. 769-781, 2011.
[20] Y. Zhou and H. Zhuang, "The optimization of lane assignment and signal timing at the tandem intersection with pre-signal," Journal of Advanced Transportation, vol. 48, no. 4, pp. 362-376, 2014.

[21] J. Long, Z. Gao, H. Zhang, and W. Y. Szeto, "A turning restriction design problem in urban road networks," European Journal of Operational Research, vol. 206, no. 3, pp. 569-578, 2010.

[22] X. Guang and L. Wu, "A model of the urban road intersection left-turning restriction," Journal of Theoretical and Applied Information Technology, vol. 48, no. 3, pp. 1619-1625, 2013.

[23] J. Long, W. Y. Szeto, and H.-J. Huang, "A bi-objective turning restriction design problem in urban road networks," European Journal of Operational Research, vol. 237, no. 2, pp. 426-439, 2014.

[24] L. R. Foulds, D. C. Duarte, H. A. do Nascimento, H. J. Longo, and B. R. Hall, "Turning restriction design in traffic networks with a budget constraint," Journal of Global Optimization, vol. 60, no. 2, pp. 351-371, 2014.

[25] F. V. Webster, “Traffic signal settings," Technical Report Road Research Technical Paper, No. 39, Road Research Laboratory, London, UK, 1958.

[26] HBS, Handbuch für die Bemessung von Straßenverkehrsanlagen (HBS, German Highway Capacity Manual), Road and Transport Association, Cologne, Germany, 2001.

[27] K. B. Kesur, "Optimization of mixed cycle length traffic signals," Journal of Advanced Transportation, vol. 48, no. 5, pp. 431-442, 2014.

[28] M. Di Gangi, G. E. Cantarella, R. D. Pace, and S. Memoli, "Network traffic control based on a mesoscopic dynamic flow model," Transportation Research Part C: Emerging Technologies, vol. 66, pp. 3-26, 2016.

[29] S. Memoli, G. E. Cantarella, S. de Luca, and R. D. Pace, "Network signal setting design with stage sequence optimisation," Transportation Research Part B: Methodological, vol. 100, pp. 20-42, 2017.

[30] G. Improta and G. E. Cantarella, "Control system design for an individual signalized junction," Transportation Research Part B: Methodological, vol. 18, no. 2, pp. 147-167, 1984.

[31] J. P. Silcock, "Designing signal-controlled junctions for groupbased operation," Transportation Research Part A: Policy and Practice, vol. 31, no. 2, pp. 157-173, 1997.

[32] X. Ma, J. Jin, and W. Lei, "Multi-criteria analysis of optimal signal plans using microscopic traffic models," Transportation Research Part D: Transport and Environment, vol. 32, pp. 1-14, 2014.

[33] C. K. Wong and S. C. Wong, "Lane-based optimization of signal timings for isolated junctions," Transportation Research Part B: Methodological, vol. 37, no. 1, pp. 63-84, 2003.

[34] C. K. Wong and B. G. Heydecker, "Optimal allocation of turns to lanes at an isolated signal-controlled junction," Transportation Research Part B: Methodological, vol. 45, no. 4, pp. 667-681, 2011.

[35] Y. Liu and C. K. Wong, "Refining Lane-Based Traffic Signal Settings to Satisfy Spatial Lane Length Requirements," Journal of Advanced Transportation, vol. 2017, Article ID 8167530, 27 pages, 2017.

[36] J. Zhao, W. Ma, K. L. Head, and X. Yang, "Dynamic turning restriction management for signalized road network," Transportation Research Record, vol. 2487, pp. 96-111, 2015.

[37] J. Zhao, Y. Liu, and P. Li, "A network enhancement model with integrated lane reorganization and traffic control strategies," Journal of Advanced Transportation, vol. 50, no. 6, pp. 1090-1110, 2016. 
[38] T. Pohlmann, New Approaches for Online Control of Urban Traffic Signal Systems [Ph.D. thesis], Fakultaet Architektur, Bauingenieurwesen und Umweltwissenschaften der Technischen Carolo-Wilhelmina zu Braunschweig, 2010.

[39] Q. Tang and B. Friedrich, "Minimization of Travel Time in Signalized Networks by Prohibiting Left Turns," Transportation Research Procedia, vol. 14, pp. 3446-3455, 2016.

[40] R. Akcelik, "Traffic signals: capacity and timing analysis," Technical Report Research report ARR No. 123, Australian Road Research Board, 1981.

[41] HCM, Highway Capacity Manual. Chapter 16: Signalized Intersections, Transportation Research Board (TRB), Washington, DC, USA, 2000.

[42] RiLSA. Richtlinien für Lichtsignalanlagen (RiLSA, Guidelines for Traffic Signals). Technical Report, Cologne, Germany, 2015.

[43] K. Meffert and N. Rotstan, Jgap: Java genetic algorithm package. 2015, http://jgap.sourceforge.net/.

[44] Y. Sheffi, Urban transportation networks, Prentice Hall, 1985.

[45] A. Hajbabaie, J. C. Medina, and R. F. Benekohal, "Effects of ITS-Based Left Turn Policies on Network Performance," in Proceedings of the 13th International IEEE Conference on Intelligent Transportation Systems, ITSC 2010, pp. 80-84, September 2010. 


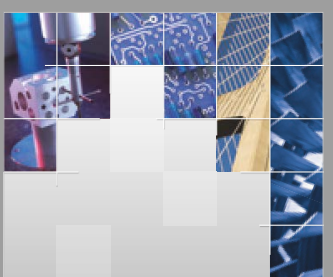

\section{Enfincering}
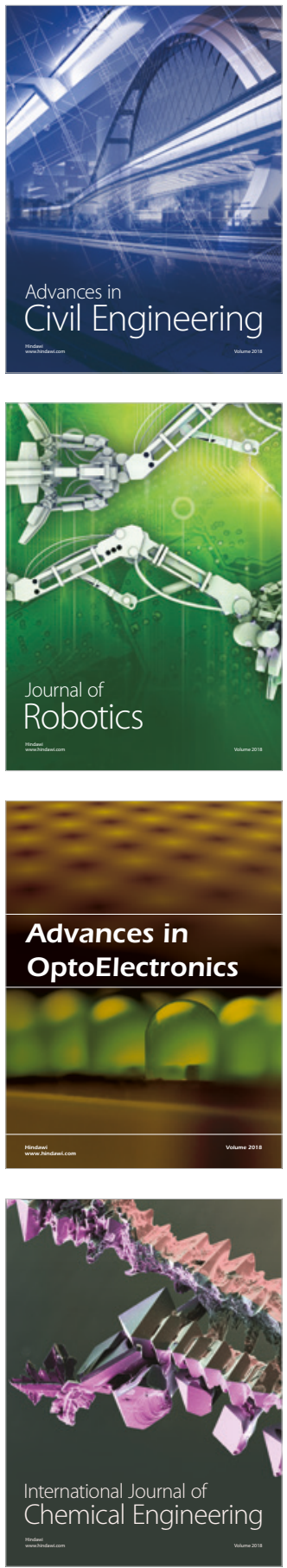

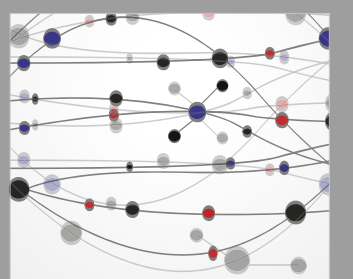

\section{Rotating \\ Machinery}

The Scientific World Journal

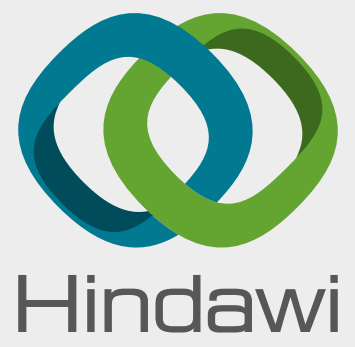

Submit your manuscripts at

www.hindawi.com
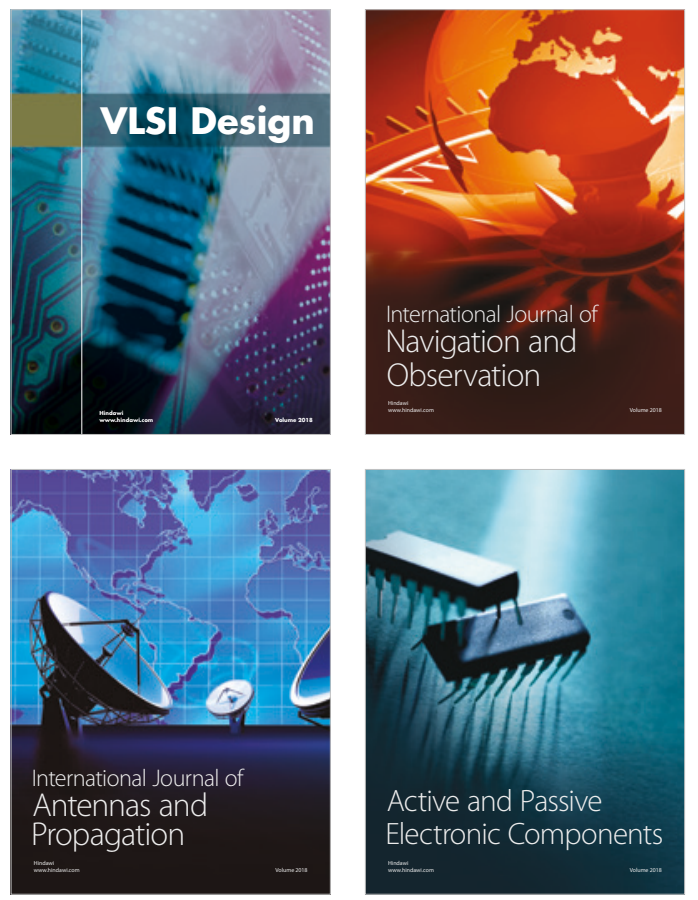
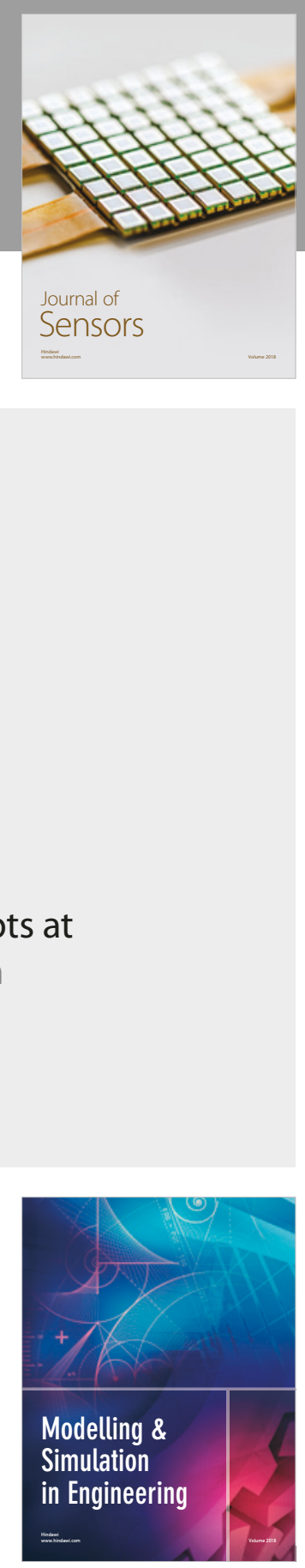

\section{Advances \\ Multimedia}
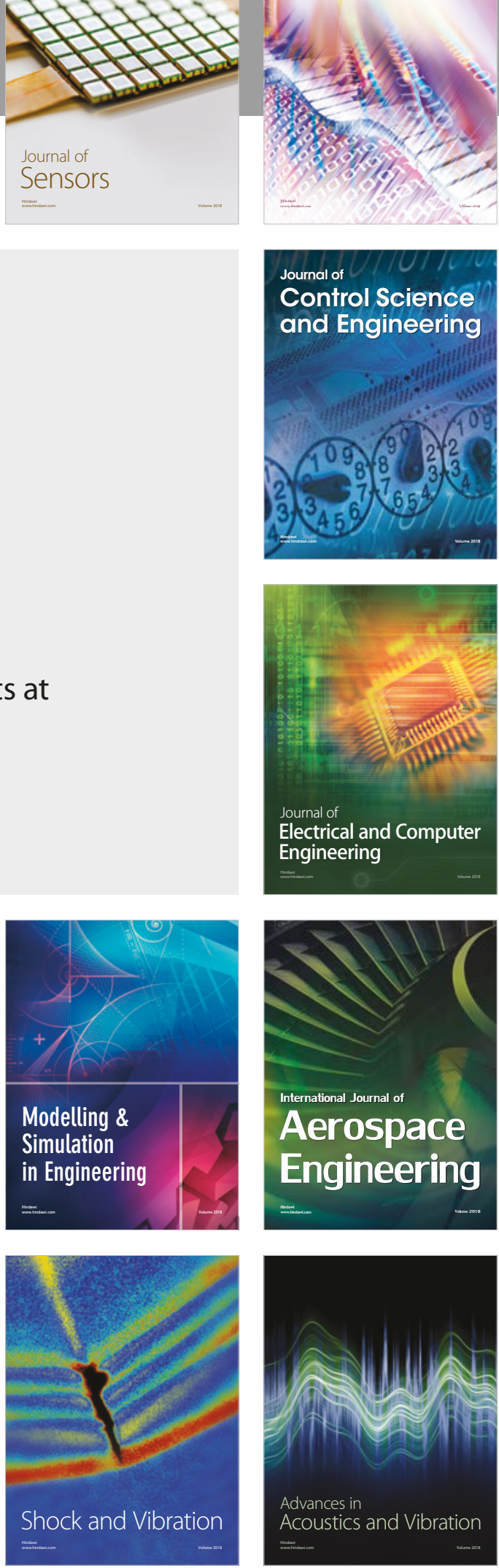